\title{
Mitochondrial DNA sequence variation and neurodegeneration
}

\author{
Michelangelo Mancuso," Massimiliano Filosto, Daniele Orsucci and Gabriele Siciliano \\ Department of Neuroscience, Neurological Clinic, University of Pisa, Via Roma 67, 56126 Pisa, Italy, and Neurological Institute, \\ University of Brescia, Section for Neuromuscular Diseases and Neuropathies, University Hospital Spedali Civili, Piazza Spedali Civili 1, \\ 25100 Bresia, Italy \\ *Correspondence to: Tel: +39050 992440; Fax: +39050 554808; E-mail: mmancuso@inwind.it
}

Date received (in revised form): 7th March, 2008

\begin{abstract}
Mitochondria, the powerhouse of the cell, play a critical role in several metabolic processes and apoptotic pathways. Many lines of evidence suggest that mitochondria have a central role in ageing-related neurodegenerative diseases. Moreover, there is a long history of investigations on mitochondria aimed at identifying genetic markers relating to ageing and neurodegenerative diseases. In this review, some of the major neurodegenerative disorders are highlighted and the role of mitochondrial haplogroups in the pathogenetic cascade leading to these diseases is discussed.
\end{abstract}

Keywords: mitochondria, mtDNA, mtDNA haplogroups, neurodegenerative disorders

\section{Introduction}

Mitochondria and their DNA (mitochondrial DNA [mtDNA]) result from a process of endosymbiosis which occurred about 1.5 billion years ago, when protobacteria populated primordial eukaryotic cells and took permanent residence in the new environment. MtDNA - a relic but not a fossil - has lost much of its independence but keeps functioning under the overarching control of the nuclear genome (nDNA).

Mitochondria are ubiquitous in eukaryotes and are essential for survival. Their primary function is to support aerobic respiration and to provide energy and heat. Mitochondria also play other important roles, including in cell signalling for apoptotic cell death.

By convention, the term 'mitochondrial diseases' refers to disorders of the mitochondrial respiratory chain, thus excluding dysfunction in other metabolic pathways located in the mitochondria (ie pyruvate metabolism, Krebs cycle and fatty acid oxidation). The respiratory chain is the only metabolic pathway in the cell that is under the dual control of mtDNA and nDNA.
There is a long history of investigations on mitochondria, which have been aimed at identifying genetic markers relating to ageing, neuromuscular and neurodegenerative diseases and, more recently, common diseases such as diabetes and cancer.

This paper will include a brief section on mitochondrial biology and genetics, and will then focus on the role of mtDNA haplogroups in selected neurodegenerative disorders.

\section{Mitochondrial compartment}

Mitochondria are highly dynamic and pleomorphic organelles. They are composed of a smooth outer membrane surrounding an inner membrane of significantly larger surface area, which, in turn, surrounds a protein-rich core - the matrix. ${ }^{1}$ Although mitochondria contain their own genome and protein-synthesising machinery, the majority of mitochondrial polypeptides are encoded in the nuclear genome, synthesised in the cytosol and imported into the mitochondria post-transcriptionally.

The main mitochondrial role is in the synthesis of ATP formed by oxidative phosphorylation. They 
are also involved in other metabolic processes, including the biosynthesis of amino acids, vitamin cofactors, fatty acids, iron-sulphur clusters, cell signalling and programmed cell death. ATP molecules are generated via glycolysis or by oxidation of glucose to ethanol or lactic acid. Electrons from oxidative substrates are transferred to oxygen, via a series of redox reactions, to generate water. In the process, protons are pumped from the matrix across the mitochondrial inner membrane via the electron transport chain (ETC), which consists of four multimeric complexes (I to IV) plus two small electron carriers - coenzyme Q (also known as ubiquinone) and cytochrome $c$. This process creates an electrochemical proton gradient. ATP is produced by the influx of these protons back through the complex V, or ATP synthase (the 'rotary motor'). ${ }^{2}$

\section{Mitochondrial genome and common mtDNA haplogroups}

Mitochondria contain two to ten molecules of mtDNA. It is a circular molecule of 16.5 kilobases lacking introns, and consisting of a heavy chain $(\mathrm{H})$ and a light chain (L). ${ }^{1,3}$ MtDNA carries 37 genes encoding 22 transfer RNAs (tRNAs), two ribosomal RNAs (12S and 16S) and 13 polypeptides (mRNAs). The $13 \mathrm{mtDNA}$-encoded polypeptides are part of the respiratory chain system and are assembled together with nuclear-encoded subunits. Seven of these belong to complex I or reduced nicotinamide adenine dinucleotide (NADH) dehydrogenase - NADH, ubiquinone oxidoreductase (ND1, ND2, ND3, ND4, ND4L, ND5, ND6). One belongs to complex III or ubiquinol - ferricytochrome c oxidoreductase. Three belong to complex IV or cytochrome c oxidase - COX I, COX II and COX III. The final two belong to complex V or ATP synthase - ATPase6 and ATPase8. The remaining mitochondrial proteins, including all of the complex II subunits, are encoded by nDNA.

In humans, mtDNA is transmitted through the maternal lineage. ${ }^{1,3}$ MtDNA is commonly used to complement information provided by $\mathrm{Y}$ chromosome studies on the evolution of modern humans.
We can theoretically follow the transmission of mtDNA from the original 'ancestor mother' by identifying common polymorphisms that have accumulated with time. Specific and unique sets of those common polymorphisms define groups of mtDNA, named haplogroups, that have evolved from the same ancestor. Phylogeographic studies identified the first common ancestor, named 'mitochondrial Eve', 4 postulated to have lived in Africa about 200,000 years ago. Direct sequencing and restriction fragment length polymorphism analysis enabled the identification of the mtDNA haplogroup tree and the mtDNA migration route (Figure 1).

The basal branching structure of mtDNA variation in most parts of the world is now well understood. ${ }^{5}$ African haplogroups fall into seven major families (L0, L1, L2, L3, L4, L5 and L6). About 85,000 years ago, probably in the Horn of Africa, the root of haplogroup L3 gave rise to many descendant haplogroups (probably because of some colonisation event or local population growth). Non-African mtDNA descends from L3 and belongs either to the $M$ or $N$ superclades (obviously excluding the descendants of migrations from Africa within the past few thousand years). In the Indian subcontinent and in South-East Asia, there is the richest basal variation in the tree originated by haplogroups $M$ and $N$, and this suggests a rapid colonisation along the southern coast of Asia about 60,000 years ago. ${ }^{5}$ The expansions northward occurred later, about 45,000 years ago. Over 30 subclades of haplogroup $M$ are present in Asia. Haplogroups A, B, C, D and X have been found in the Americas, although come mainly from Asia.

In Europeans and Near Easterners (who share a rather recent common ancestor), nine different mitochondrial haplogroups have been identified $(\mathrm{H}, \mathrm{I}, \mathrm{J}, \mathrm{K}, \mathrm{T}, \mathrm{U}, \mathrm{V}, \mathrm{W}$ and $\mathrm{X})$. The variation in the basal European mtDNA haplogroups dates to about 45,000 years ago. ${ }^{5}$ Complete mtDNA sequencing and the increasing number of samples analysed allow haplogroups to be subdivided into smaller groups, identifying younger branches on the mtDNA evolutionary tree. Therefore, subhaplogroup classification is continuously evolving. ${ }^{5}$ 


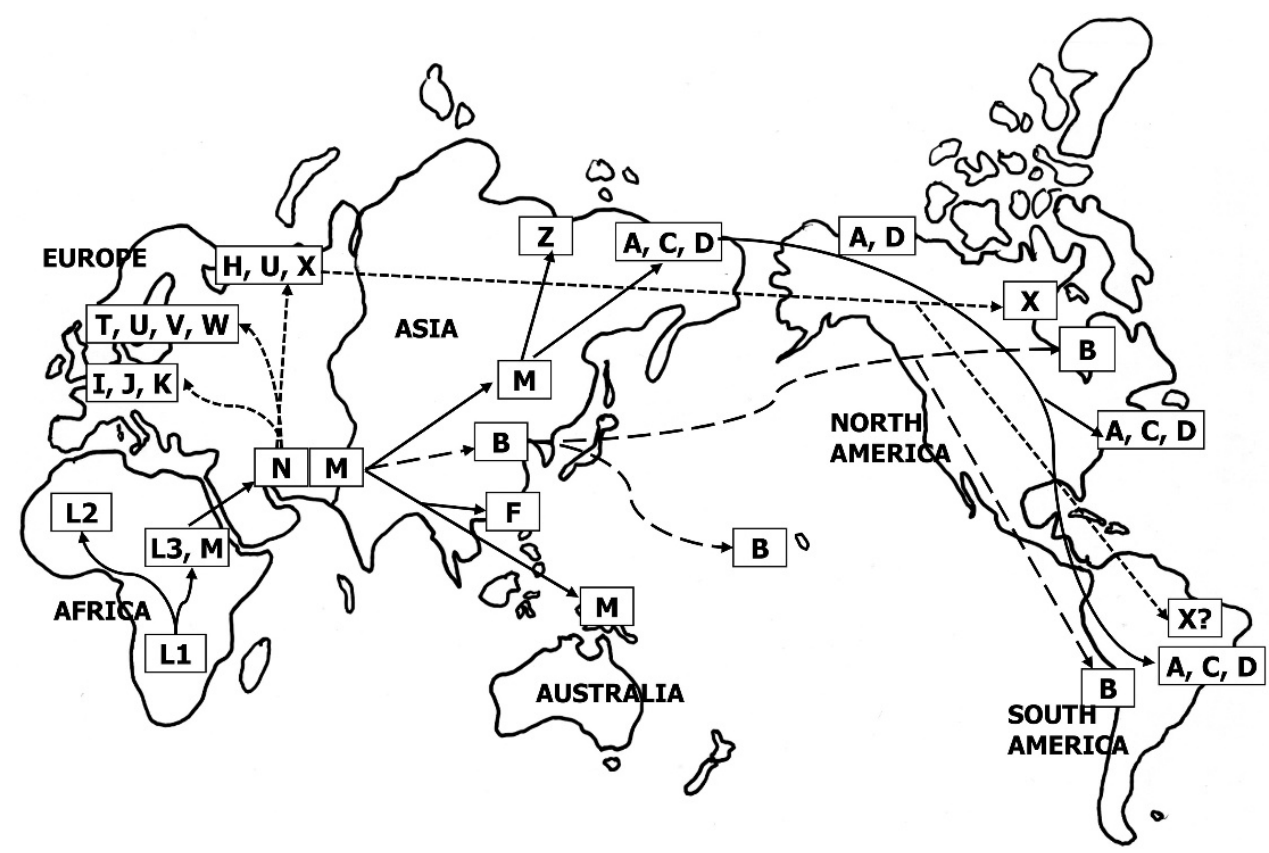

Figure I. Mitochondrial DNA (MtDNA) haplogroup migration patterns (modified from Shriver and Kittles). The map shows the migration patterns of the main mtDNA haplogroups. The first common ancestor is postulated to have lived in Africa about 200,000 years ago. From Africa, the arrows show the mtDNA migration routes. ${ }^{62}$

\section{MtDNA haplogroups and neurodegeneration}

It has been speculated that mtDNA mutations that accumulate with age might lead to impaired energy generation and to increased numbers of reactive oxygen species (ROS), both resulting in cell damage. Polymorphisms in mtDNA may cause subtle differences in the encoded proteins and, thus, minimal changes in mitochondrial respiratory chain activity and free radical overproduction. This could predispose an individual, or a population sharing the same mtDNA genotype, to an earlier onset of apoptotic processes, such as accumulation of somatic mtDNA mutations and mitochondrial impairment. The opposite could be true for different polymorphism(s), which could be beneficial, increasing mitochondrial respiration and/or reducing ROS production. ${ }^{6,7}$

Specific mitochondrial haplogroups have been linked to longevity. ${ }^{8,9}$ It therefore follows that the same or other haplogroups could be involved at the other end of the life spectrum, neurodegeneration and, thus, death. Because of the sensitivity of
mtDNA as a marker for human migration patterns, all studies of mtDNA haplogroup association with disease must pay rigorous attention to the ethnic matching of cases to controls.

\section{Alzheimer's disease}

Alzheimer's disease (AD) is a late-onset progressive neurodegenerative disorder which results in irreversible loss of neurones, particularly in the cortex and hippocampus. It is the most common form of dementia in the elderly and is clinically characterised by an impairment of cognitive function and changes in behaviour and personality. Apart from the neuronal loss, the pathological hallmarks are extracellular senile plaques containing the peptide beta-amyloid $(A \beta)$, and neurofibrillary tangles composed of a hyperphosphorylated form of the microtubular protein, tau. ${ }^{10}$ The $A \beta$ cascade hypothesis remains the main pathogenetic model of familial AD with mutations in amyloid precursor protein and presenilin genes, ${ }^{11}$ but its role in the majority of sporadic AD cases without mutations in these genes (accounting for 90-95 per cent of $\mathrm{AD}$ 
cases) is still unclear. There is substantial evidence of morphological, biochemical and genetic abnormalities in mitochondria in various tissues of patients with $\mathrm{AD}{ }^{12,13}$ For example, studies have reported a decrease in cyclo-oxygenase (COX) activity in the hippocampus and platelets of sporadic AD patients. ${ }^{14,15}$ Further, cybrid models, obtained by transfecting mtDNA from the platelets of $\mathrm{AD}$ patients into neuronal cultured cells deprived of their own mtDNA, revealed decreased COX activity, increased ROS generation, increased $\mathrm{A} \beta$ production and morphological abnormalities. ${ }^{16,17}$ This suggests a mitochondrial genomic contribution to mitochondrial dysfunction in AD. ${ }^{6,18}$ Studies attempting to identify mtDNA mutations in the brains of AD patients have had limited success. For example, Elson et al. ${ }^{19}$ sequenced the complete coding regions of 145 autoptic AD brain samples and 128 normal controls; they observed that for both synonymous and non-silent changes, the overall numbers of nucleotide substitutions were the same for the AD and control sequences. ${ }^{19}$

For the reasons described above, different groups have analysed the frequencies of polymorphisms and/or mutations in mtDNA in AD patients with conflicting results. Chagnon and coworkers ${ }^{20}$ reported that haplogroup $\mathrm{T}$ is under-represented in AD patients, while haplogroup J seems to be overrepresented. By studying a sample of Italian subjects, Carrieri et al. ${ }^{21}$ found that haplogroups $\mathrm{K}$ and $U$ were present at a lower frequency in $\mathrm{AD}$ patients who were apolipoprotein (Apo) E4 carriers than in non-carriers (while in controls there was no association between the E4 allele and mtDNA haplogroups). This suggests that $\mathrm{K}$ and $\mathrm{U}$ may act by neutralising the effect of the major AD risk factor - the E4 allele. $^{21}$ The same authors detected a lowering of the $E 4$ allele odds ratio from statistically significant to non-significant in patients with haplogroups $\mathrm{K}$ and $\mathrm{U} .^{21}$

Another report showed (independently from ApoE genotype) that males classified as haplogroup $\mathrm{U}$ had a significantly increased risk of AD, while females demonstrated a significantly decreased risk with the same $U$ haplogroup. ${ }^{22}$ Thus, the inheritance of haplogroup $U$ may have a negative effect on ageing in Caucasian males. ${ }^{22}$ Two recent studies (including only neuropathologically proven cases of $\mathrm{AD}$ in patients of European descent) indicated that mtDNA haplogroups were not associated with $\mathrm{AD}$, either individually or by grouping together closely related haplogroups. ${ }^{19,23}$

In another study, no evidence was found for an aetiological role of haplogroup-associated polymorphisms. ${ }^{24}$ In this study, the frequency of European mtDNA haplogroups was investigated in a clinically well defined group of 209 unrelated patients and 191 controls, both of clear Tuscan origin (in order to minimise the risk of false associations between gene markers and disease). The frequency of haplogroups H, I, J, K, T, U, V, W and $\mathrm{X}$ was not significantly different between the patients and control groups. ${ }^{24}$ Further, there was no significant difference between genders in mtDNA haplogroup distribution in either $\mathrm{AD}$ patients or control groups. The ApoE4 allele was confirmed as a risk factor for $\mathrm{AD}$, as it was found at a significantly higher frequency in patients than in controls (23.3 per cent versus 8.11 per cent), but no association between ApoE alleles and mtDNA haplogroups was observed. ${ }^{24}$ The data from this study also excluded any association between mtDNA haplogroups, age of onset and mean survival.

In conclusion, although it has been suggested that inherited haplogroups $\mathrm{K}$ and $\mathrm{U}$ may influence $A D$ risk in Caucasians, this is still an unresolved question. To date, mtDNA haplogroups do not seem to play a major role in $\mathrm{AD}$.

\section{Parkinson's disease}

Parkinson's disease (PD) is a common neurodegenerative movement disorder characterised by resting tremor, rigidity and bradykinesia. It is characterised by loss of dopaminergic neurones in the substantia nigra and Lewy bodies in the remaining neurones. PD exists in both familial and idiopathic forms. While the aetiology of sporadic PD remains largely unclear, there is accumulating evidence suggesting that mitochondrial dysfunction occurs in the brain and peripheral tissues of PD patients. ${ }^{25,26}$ In 
selected cases, mitochondrial genetic abnormalities can directly cause PD. ${ }^{25,26}$ Cells fused with mtDNA from PD patients showed 25 per cent decreased complex I activity. ${ }^{27}$ Parkinsonism has been associated with large-scale rearrangements in mtDNA. ${ }^{28,29}$ Further, the co-existence of parkinsonism and mutations in the mitochondrial polymerase gamma nDNA gene (POLG1) was recently described in several families, suggesting that when defective, this gene could be responsible for some of the Mendelian transmission of parkinsonism. ${ }^{30,31}$ Clustering of rare variants of the POLG1 CAG-repeat (encoding a polyglutamine tract) has been found in Finnish patients with idiopathic PD, compared to controls. ${ }^{32}$

By contrast, primary mtDNA point mutations causing parkinsonism have been reported only rarely - typically as one feature of a larger syndrome, as in the case of sensorineural deafness and neuropathy. ${ }^{33}$ Very recently, Horvath et al. ${ }^{34}$ observed the A8344G 'MERRF' (myoclonic epilepsy with ragged red fibres) mutation on the tRNALys gene of the mtDNA in a 66-year-old man with dopa-responsive parkinsonism, reduced muscle strength and ragged red fibres. No other clinical signs typical of MERRF were noted. ${ }^{34}$ The A8344G mutation was present in a virtually homoplasmic state in the patient's muscle, and 80 per cent mutant mtDNA was detected in blood DNA. ${ }^{34}$ In order to evaluate if this mutation can represent a common cause of sporadic PD, 159 Italian patients with PD were analysed; ${ }^{35}$ none of the patients carried the A8344 mutation in DNA extracts from peripheral blood lymphocytes. Thus, the screening for this mutation would have been restricted to PD patients with other canonical features of mitochondrial encephalomyopathies (ie myopathy, ophthalmoplegia, neuropathy, cerebellar ataxia), and without POLG1 mutations. ${ }^{35}$

The role of the mtDNA variants in PD has been extensively studied. Van der Walt and coworkers ${ }^{36}$ genotyped ten single nucleotide polymorphisms (SNPs) that define the European mtDNA haplogroups in 609 white patients with PD and 340 unaffected white control subjects. These authors observed that haplotypes $\mathrm{J}$ and $\mathrm{K}$ reduced the incidence of PD by 50 per cent. ${ }^{36}$ Further analysis revealed that the SNP at 9055A of ATP6 (which defines haplogroup $\mathrm{K}$ ) reduced the risk in women, and that the SNP at 13708A of the ND5 gene (haplogroup J) was protective in individuals older than 70 years (probably either through increasing the performance of complex I within the brain and other tissues in individuals derived from $\mathrm{J}$ and $\mathrm{K}$ haplogroup lineages, or reducing ROS generation). ${ }^{36}$ Consistent with the described study, it has been reported that the haplotype cluster UKJT was associated with a 22 per cent reduction in population-attributable risk for $\mathrm{PD}^{37}$ Further, Autere et al. ${ }^{38}$ hypothesised that the risk of PD is conveyed by the total number of non-synonymous substitutions in the complex I genes in various mtDNA lineages. To test this possibility, they determined the number of non-synonymous substitutions of the seven complex I genes in a Finnish population, and observed that the supercluster JTIWX increased the risk of both PD and PD with dementia. This cluster was associated with a twofold increase in non-synonymous substitutions in the mtDNA genes encoding complex I subunits. ${ }^{38}$

Another group evaluated the distribution of the different mtDNA haplogroups in a large cohort of 620 Italian patients with adult-onset idiopathic PD versus two groups of ethnic-matched controls. These authors found that haplogroup K was associated with a lower risk of PD; they also reported that the $10398 \mathrm{G}$ polymorphism was protective against PD. ${ }^{39}$ This last finding (but not the association between haplogroup $\mathrm{K}$ and lower risk) was later confirmed in 271 Spanish PD patients versus 230 healthy controls. ${ }^{40}$

\section{Amyotrophic lateral sclerosis}

Amyotrophic lateral sclerosis (ALS) is a motor neurone disease with selective degeneration of the anterior horn cells of the spinal cord and cortical motor neurones. Approximately 90 per cent of cases are sporadic and 10 per cent are familial. About 20 per cent of familial cases result from mutations in the gene encoding for $\mathrm{Cu} / \mathrm{Zn}$ 
superoxide dismutase (SOD1). The aetiology and pathogenesis of the sporadic form of the disease are poorly understood; mitochondrial dysfunction and oxidative stress are probably involved. ${ }^{6,7}$ ALS cybrids seem to have impaired respiratory chain function, increased ROS production and altered calcium homeostasis, suggesting the presence of a primary mtDNA defect, ${ }^{41}$ but these findings have not been confirmed. ${ }^{42}$ Further, increased levels of mtDNA single 'common deletion' and multiple deletions have been detected in the brain $^{43}$ and skeletal muscle ${ }^{44}$ of patients with sporadic ALS.

Three patients with ALS and primary pathogenic mtDNA mutations have been reported. The first was an out-of-frame mutation (leading to premature termination of the translation) of mtDNA encoding for subunit I of COX, discovered during investigation of a severe isolated muscle COX deficiency in a patient with early-onset motor neurone-like degeneration, but without the common features of mitochondrial disorders. ${ }^{45}$ The second was a homoplasmic mutation in the mitochondrial $t R N A C y s$, in a 60-year-old Caucasian male suffering from an asymmetrical pure lower motor neurone variant of ALS and temporal lobe epilepsy. ${ }^{46}$ The third was a point mutation in the tRNAIle (T4274C), in a patient with pure lower motor neurone disease, diabetes and cardiac involvement. ${ }^{47}$

To investigate if specific genetic polymorphisms within the mtDNA could act as susceptibility factors and contribute to the clinical expression of sporadic ALS, predefined European mtDNA haplogroups were genotyped in 222 patients of clear Italian origin with sporadic ALS and 151 matched controls. ${ }^{48}$ Mutations on the entire SOD1 gene were excluded by single strand conformation polymorphism analysis and/or by direct sequencing. The frequency of haplogroup I was found to be lower in ALS cases than in controls (odds ratio [OR] 0.08, 95\% confidence interval [CI] 0.04$0.40, \quad p<0.01){ }^{48}$ Multiple regression studies which aimed to test the hypothesis that mtDNA haplogroups could influence the age of onset, severity and neurological system involved in the disease did not reach significance. In a comparison developed to investigate how haplogroup I differs from the other haplogroups tested, two nucleotides at positions 16391 and 10034 were identified; only the difference between $16391 \mathrm{~A}$ and $10034 \mathrm{C}$ alleles was found to be highly significant $(p<0.01) .{ }^{48}$ In accordance with the described study, mtDNA polymorphism might contribute to motor neurone degeneration, possibly interacting with unknown genetic or environmental factors. ${ }^{48}$ This finding was not confirmed in a recent paper by Chinnery et al., ${ }^{49}$ who studied a UK cohort of 504 ALS patients and 493 controls and found no evidence that mtDNA haplogroups contribute to the risk of developing ALS.

\section{Multiple sclerosis}

Multiple sclerosis (MS) is a chronic inflammatory autoimmune disease, with myelin loss and gliosis, affecting young people — mostly females. Family-based half-sibling studies suggest that there is a maternal parent-of-origin effect in MS. ${ }^{50}$ The involvement of mtDNA in determining susceptibility to MS has been hypothesised based on the detection of Leber's hereditary optic neuropathy (LHON) mutations in MS patients more frequently than expected by chance. ${ }^{51-55}$ In a recent study, 21 of the 58 patients $(36.2$ per cent) tested positive for the T4216C mutation, while only 11.3 per cent of the controls carried this secondary LHON mutation $(p<0.01) .{ }^{56}$ In a study with 77 Caucasian MS patients versus 84 controls, haplogroups $\mathrm{K}$ and $\mathrm{J}$ showed an association with $\mathrm{MS}$ at a $p$-value of $0.001 .^{57}$

Two recent papers reported that a SNP (RS660339) in the UCP2 nuclear gene was a risk factor for MS. ${ }^{58,59}$ UCP2 is a member of the mitochondrial proton transport family, which uncouples proton entry in the mitochondrial matrix from ATP synthesis. The RS660339 SNP is correlated with lower UCP2 expression levels in vitro and in vivo. ${ }^{58}$ Low levels of UCP2 are a risk factor for MS. In the second study, ${ }^{59}$ the authors also found that none of the mitochondrial haplogroups were associated with the disease; however, there seemed to be a protective trend in the ' $\mathrm{JT}+$ the protective allele of UCP2' combination. ${ }^{59}$ These genetic 
variables are independently hereditary, but there may be a synergetic effect between them.

Very recently, $\mathrm{Yu}$ and co-workers ${ }^{60}$ investigated more than 2,500 sporadic cases of MS and a similar number of healthy controls. They studied seven common SNPs in the mtDNA, and found that one of these SNPs (nt13708 G/A) was significantly associated with $\mathrm{MS}$ susceptibility $(\mathrm{OR}=1.71,95$ per cent CI 1.28-2.26, $p=0.0002) .{ }^{60}$ The frequency of haplogroup $\mathrm{J}$, a haplogroup comprising nt10398 and nt13708 SNPs, was higher in patients than in controls. ${ }^{60}$ Subsequent sequencing of the mtDNA of 50 individuals revealed that the nt13708A variant itself, rather than the SNPs linked to it, was responsible for the association. ${ }^{60}$ Thus, this mtDNA variant might represent a susceptibility allele for MS.

\section{Conclusions}

In the past 15 years, research has been directed at clarifying the involvement of mitochondria, and defects in mitochondrial oxidative phosphorylation, in late-onset neurodegenerative disorders. A critical role for mitochondrial dysfunction and oxidative damage in neurodegenerative diseases has been greatly strengthened by recent findings. Despite the evidence of morphological and biochemical abnormalities of the mitochondria in various tissues of patients with neurodegenerative disorders, however, the role of the mitochondrial genome and its haplogroups as a risk factor is still controversial. MtDNA haplogroups have been associated with a number of different neurodegenerative diseases, but, to date, the only disease consistently associated with a different mtDNA haplogroup frequency is PD. Because very large cohorts are required reliably to detect an association with complex human diseases, ${ }^{61}$ further analysis, using larger samples, will be required to reveal more definitively the contribution of mtDNA mutations or haplogroups, if any, to the pathogenesis of neurodegenerative diseases.

\section{Acknowledgments}

The authors are grateful to Dr Anna Choub for her critical reading of the manuscript, and to Dr Lucia Petrozzi for preparing Figure 1.

\section{References}

1. DiMauro, S. and Schon, E.A. (2003), 'Mitochondrial respiratory-chain diseases', N. Engl. J. Med. Vol. 348, pp. 2656-2668.

2. Noji, H. and Yoshida, M. (2001), 'The rotary machine of the cell, ATP synthase', J. Biol. Chem. Vol. 276, pp. 1665-1668.

3. Filosto, M. and Mancuso, M. (2007), 'Mitochondrial diseases: A nosological update', Acta Neurol. Scand. Vol. 115, pp. 211-221.

4. Cann, R.L., Stoneking, M. and Wilson, A.C. (1987), 'Mitochondrial DNA and human evolution', Nature Vol. 325, pp. 31-36.

5. Torroni, A., Achilli, A., Macaulay, V. et al. (2006), 'Harvesting the fruit of the human mtDNA tree', Trends Genet. Vol. 22, pp. 339-345.

6. Petrozzi, L., Ricci, G., Figlioli, N.J. et al. (2007), 'Mitochondria and neurodegeneration', Biosci. Rep. Vol. 27, pp. 87-104.

7. Mancuso, M., Coppede, F., Migliore, L. et al. (2006), 'Mitochondrial dysfunction, oxidative stress and neurodegeneration', J. Alzheimers Dis. Vol. 10, pp. 59-73.

8. Zhang, J., Asin-Cayuela, J., Fish, J. et al. (2003), 'Strikingly higher frequency in centenarians and twins of mtDNA mutation causing remodeling of replication origin in leukocytes', Proc. Natl. Acad. Sci. USA Vol. 100, pp. 1116-1121.

9. Niemi, A.K., Moilanen, J.S., Tanaka, M. et al. (2005), 'A combination of three common inherited mitochondrial DNA polymorphisms promotes longevity in Finnish and Japanese subjects', Eur. J. Hum. Genet. Vol. 13, pp. $166-170$.

10. Selkoe, D.J. (2001), 'Alzheimer's disease: Genes, proteins, and therapy', Physiol. Rev. Vol. 81, pp. 741-766.

11. Hardy, J. and Selkoe, D.J. (2002), 'The amyloid hypothesis of Alzheimer's disease: Progress and problems on the road to therapeutics', Science Vol. 297, pp. 353-356

12. Swerdlow, R.H. and Khan, S.M. (2004), 'A "mitochondrial cascade hypothesis" for sporadic Alzheimer's disease', Med. Hypotheses Vol. 63, pp. 8-20.

13. Beal, M.F. (2005), 'Mitochondria take center stage in aging and neurodegeneration', Ann. Neurol. Vol. 58, pp. 495-505.

14. Bosetti, F., Brizzi, F., Barogi, S. et al. (2002), 'Cytochrome c oxidase and mitochondrial F1F0-ATPase (ATP synthase) activities in platelets and brain from patients with Alzheimer's disease', Neurobiol. Aging Vol. 23, pp. $371-376$.

15. Mancuso, M., Filosto, M., Borsetti, F. et al. (2003), 'Decreased platelet cytochrome $\mathrm{c}$ oxidase activity is accompanied by increased blood lactate concentration during exercise in patients with Alzheimer disease', Exp. Neurol. Vol. 182, pp. 421-426.

16. Khan, S.M., Cassarino, D.S., Abramova, N.N. et al. (2000), 'Alzheimer's disease cybrids replicate beta-amyloid abnormalities through cell death pathways', Ann. Neurol. Vol. 48, pp. 148-155.

17. Trimmer, P.A., Keeney, P.M., Borland, M.K. et al. (2004), 'Mitochondrial abnormalities in cybrid cell models of sporadic Alzheimer's disease worsen with passage in culture', Neurobiol. Dis. Vol. 15, pp. 29-39.

18. Onyango, I., Khan, S., Miller, B. et al. (2006), 'Mitochondrial genomic contribution to mitochondrial dysfunction in Alzheimer's disease', J. Alzheimers Dis. Vol. 9, pp. 183-193.

19. Elson, J.L., Herrnstadt, C., Preston, G. et al. (2006), 'Does the mitochondrial genome play a role in the etiology of Alzheimer's disease?', Hum. Genet. Vol. 119, pp. 241-254.

20. Chagnon, P., Gee, M., Filion, M. et al. (1999), 'Phylogenetic analysis of the mitochondrial genome indicates significant differences between patients with Alzheimer disease and controls in a French-Canadian founder population', Am. J. Med. Genet. Vol. 85, pp. 20-30.

21. Carrieri, G., Bonafe, M., De Luca, M. et al. (2001), 'Mitochondrial DNA haplogroups and ApoE4 allele are non-independent variables in sporadic Alzheimer's disease', Hum. Genet. Vol. 108, pp. 194-198.

22. Van der Walt, J.M., Dementieva, Y.A., Martin, E.R. et al. (2004), 'Analysis of European mitochondrial haplogroups with Alzheimer disease risk', Neurosci. Lett. Vol. 365, pp. 28-32.

23. Chinnery, P.F, Taylor, G.A., Howell, N. et al. (2000), 'Mitochondrial DNA haplogroups and susceptibility to $\mathrm{AD}$ and dementia with Lewy bodies', Neurology Vol. 55, pp. 302-304. 
24. Mancuso, M., Nardini, M., Micheli, D. et al. (2007), 'Lack of association between mtDNA haplogroups and Alzheimer's disease in Tuscany', Neurol. Sci., Vol. 28, pp. 142-147.

25. Lean, M.T. and Beal, M.F. (2006), 'Mitochondrial dysfunction and oxidative stress in neurodegenerative diseases', Nature Vol. 443, pp. 787-795.

26. Fukae, J., Mizuno, Y. and Hattori, N. (2007), 'Mitochondrial dysfunction in Parkinson's disease', Mitochondrion Vol. 7, pp. 58-62

27. Gu, M., Cooper, J.M., Taanman, J.W. et al. (1998), 'Mitochondrial DNA transmission of the mitochondrial defect in Parkinson's disease', Ann. Neurol. Vol. 44, pp. 177-186.

28. Casali, C., Bonifatti, V., Santorelli, FM. et al. (2001), 'Mitochondrial myopathy, parkinsonism, and multiple mtDNA deletions in a Sephardic Jewish family', Neurology Vol. 56, pp. 802-805.

29. Siciliano, G., Mancuso, M., Ceravolo, R. et al. (2001), 'Mitochondrial DNA rearrangements in young onset parkinsonism: Two case reports', J. Neurol. Neurosurg. Psychiatry Vol. 71, pp. 685-687.

30. Davidzon, G., Greene, P., Mancuso, M. et al. (2006), 'Early-onset familial parkinsonism due to POLG mutations', Ann. Neurol. Vol. 59, pp. 859-862.

31. Luoma, P., Melberg, A., Rinne, J.O. et al. (2004), 'Parkinsonism, premature menopause, and mitochondrial DNA polymerase mutations: Clinical and molecular genetic study', Lancet Vol. 364, pp. 875-882.

32. Luoma, P., Eerola, J., Ahola, S. et al. (2007), 'Mitochondrial DNA polymerase gamma variants in idiopathic sporadic Parkinson disease', Neurology Vol. 69, pp. 1152-1159.

33. Thyagarajan, D., Bressman, S., Bruno, C. et al. (2000), 'A novel mitochondrial 12 SrRNA point mutation in parkinsonism, deafness, and neuropathy', Ann. Neurol. Vol. 48, pp. 730-736.

34. Horvath, R., Kley, R.A., Lochmuller, H. et al. (2007), 'Parkinson syndrome, neuropathy, and myopathy caused by the mutation A8344G (MERRF) in tRNALys', Neurology Vol. 68, pp. 56-58

35. Mancuso, M., Nesti, C., Petrozzi, L. et al. (2008), 'The mtDNA A8344G "MERRF" mutation is not a common cause of sporadic Parkinson disease in Italian population', Parkinsonism Relat. Disord. Vol. 14, pp. 381-382.

36. Van der Walt, J.M., Nicodemus, K.K., Martin, E.R. et al. (2003), 'Mitochondrial polymorphisms significantly reduce the risk of Parkinson's disease', Am. J. Hum. Genet. Vol. 72, pp. 804-811.

37. Pyle, A., Foltynie, T., Tiangyou, W. et al. (2005), 'Mitochondrial DNA haplogroup cluster UKJT reduces the risk of PD', Ann. Neurol. Vol. 57, pp. $564-567$.

38. Autere, J., Moilanen, J.S., Finnila, S. et al. (2004), 'Mitochondrial DNA polymorphisms as risk factors for Parkinson's disease and Parkinson's disease dementia', Hum. Genet. Vol. 115, pp. 29-35.

39. Ghezzi, D., Marelli, C., Achilli, A. et al. (2005), 'Mitochondrial DNA haplogroup $\mathrm{K}$ is associated with a lower risk of Parkinson's disease in Italians', Eur. J. Hum. Genet. Vol. 13, pp. 748-752.

40. Huerta, C., Castro, M.G., Coto, E. et al. (2005), 'Mitochondrial DNA polymorphisms and risk of Parkinson's disease in Spanish population', J. Neurol. Sci. Vol. 236, pp. 49-54.

41. Swerdlow, R.H., Parks, J.K., Cassarino, D.S. et al. (1998), 'Mitochondria in sporadic amyotrophic lateral sclerosis', Exp. Neurol. Vol. 153, pp. 135-142.

42. Gajewski, C.D., Lin, M.T., Cudkowicz, M.E. et al. (2003), 'Mitochondrial DNA from platelets of sporadic ALS patients restores normal respiratory functions in $\mathrm{rho}(0)$ cells', Exp. Neurol. Vol. 179, pp. 229-235.

43. Dhaliwal, G.K. and Grewal, R.P. (2000), 'Mitochondrial DNA deletion mutation levels are elevated in ALS brains', Neuroreport Vol. 11, pp. 2507-2509
44. Ro, L.S., Lai, S.L., Chen, C.M. et al. (2003), 'Deleted 4977-bp mitochondrial DNA mutation is associated with sporadic amyotrophic lateral sclerosis: A hospital-based case-control study', Muscle Nerve Vol. 28, pp. $737-743$.

45. Comi, G.P., Bordoni, A., Salani, S. et al. (1998), 'Cytochrome c oxidase subunit I microdeletion in a patient with motor neuron disease', Ann. Neurol. Vol. 43, pp. 110-116.

46. Kirches, E., Winkler, K., Vielhaber, S. et al. (1999), 'Mitochondrial tRNA(Cys) mutation A5823G in a patient with motor neuron disease and temporal lobe epilepsy', Pathobiology Vol. 67, pp. 214-218.

47. Borthwick, G.M., Taylor, R.W., Walls, T.J. et al. (2006), 'Motor neuron disease in a patient with a mitochondrial tRNAIle mutation', Ann. Neurol. Vol. 59, pp. 570-574.

48. Mancuso, M., Conforti, FL., Rocchi, A. et al. (2004), 'Could mitochondrial haplogroups play a role in sporadic amyotrophic lateral sclerosis?', Neurosci. Lett. Vol. 371, pp. 158-162.

49. Chinnery, P.F., Mowbray, C., Elliot, H. et al. (2007), 'Mitochondrial DNA haplogroups and amyotrophic lateral sclerosis', Neurogenetics Vol. 8, pp. 65-67.

50. Ebers, G.C., Sadovnick, A.D., Dyment, D.A. et al. (2004), 'Parent-of-origin effect in multiple sclerosis: observations in half-siblings', Lancet Vol. 363, pp. 1773-1774.

51. Flanigan, K.M. and Johns, D.R. (1993), 'Association of the 11,778 mitochondrial DNA mutation and demyelinating disease', Neurology Vol. 43, pp. $2720-2722$.

52. Harding, A.E., Sweeney, M.G., Millerb, H. et al. (1992), 'Occurrence of multiple sclerosis-like illness in women who have a Leber's hereditary optic neuropathy mitochondrial DNA mutation', Brain Vol. 115, pp. 979-989.

53. Olsen, N.K., Hansen, A.W., Norby, S. et al. (1995), 'Leber's hereditary optic neuropathy associated with a disorder indistinguishable from multiple sclerosis in a male harboring the mitochondrial DNA 11.778 mutation', Acta Neurol. Scand. Vol. 91, pp. 326-329.

54. Hanefeld, F.A., Ernst, B.P., Wilichowski, E. et al. (1994), 'Leber's hereditary optic neuropathy mitochondrial DNA mutations in childhood multiple sclerosis', Neuropediatrics Vol. 25, p. 331.

55. Kalman, B., Lublin, FD. and Alder, H. (1995), 'Mitochondrial DNA mutations in multiple sclerosis', Mult. Scler. Vol. 1, pp. 32-36.

56. Mihailova, S.M., Ivanova, I., Quin, L.M. et al. (2007), 'Mitochondrial DNA variants in Bulgarian patients affected by multiple sclerosis', Eur. J. Neurol. Vol. 14, pp. 44-47.

57. Kalman, B., Li, S., Chatterjee, D. et al. (1999), 'Large scale screening of the mitochondrial DNA reveals no pathogenic mutations but a haplotype associated with multiple sclerosis in Caucasians', Acta Neurol. Scand. Vol. 99, pp. 16-25.

58. Vogler, S., Goedde, R., Miterski, B. et al. (2005), 'Association of a common polymorphism in the promoter of UCP2 with susceptibility to multiple sclerosis', J. Mol. Med. Vol. 83, pp. 806-811.

59. Otaegui, D., Saenz, A., Ruız-Martınez, J. et al. (2007), 'UCP2 and mitochondrial haplogroups as a multiple sclerosis risk factor', Mult. Scler. Vol. 13, pp. 454-458.

60. Yu, X., Koczan, D., Sulonen, A. et al. (2008), 'mtDNA nt13708A variant increases the risk of multiple sclerosis', PLoS ONE [Internet], Vol. 3, pp. e1530. Available at: http://www.plosone.org/article/info:doi/10.1371/ journal.pone.0001530.

61. Samuels, D.C., Carothers, A.D., Horton, R. et al. (2006), 'The power to detect disease associations with mitochondrial DNA haplogroups', Am. J. Hum. Genet. Vol. 78, pp. 713-720.

62. Shriver, M.D. and Kittles, R.A. (2004), 'Genetic ancestry and the search for personalized genetic histories', Nat. Rev. Genet. Vol. 5, pp. $611-618$ 